\title{
High Performance Liquid Chromatography (HPLC): The Workhorse in the Analytical Laboratory
}

\author{
Victoria F. Samanidou* \\ Laboratory of Analytical Chemistry, Department of Chemistry, Aristotle University of Thessaloniki 54124, Thessaloniki, Greece
}

Received: September 26, 2015; Accepted: December 29, 2015; Published: December 31, 2015

*Corresponding authors: Victoria Samanidou, Laboratory of Analytical Chemistry, Department of Chemistry, Aristotle University of Thessaloniki 54124, Thessaloniki, Greece, Tel: +30231997698; Fax: +302310997719; E-mail: samanidu@chem.auth.gr

Instrumental analysis has disposed classical chemical analysis in every analytical laboratory, since many decades, as it is faster and more productive. Beyond any doubt chromatography and above all High Performance Liquid Chromatography (HPLC) is the workhorse in routine analysis, either as standalone technique or hyphenated with specific and more sensitive spectroscopic techniques. Thus the detection of analytes can be feasible at low concentrations following regulatory requirements, as for example those set by environmental protection agencies, food authorities or pharmacopeias, that are getting stricter every day.

HPLC is the analytical tool used for the elucidation of biological processes, for the detection of food adulteration, in doping control, in clinical studies, in forensics, toxicology etc.

All separation mechanisms are mobilized to cover all analytical needs. Chiral chromatography in pharmaceutical analysis is necessary to check the enantioselectivity required for chiral drugs of different activity. Affinity chromatography provides the desired separation of proteins and antibodies in the emerging class of new therapeutic agents of biopharmaceuticals. Ion exchange chromatography can be used for the separation of charged species like monoclonal antibodies with net positive charge so that under the right $\mathrm{pH}$ conditions their elution or retention can be facilitated accordingly. Size exclusion chromatography enables the separation of proteins, or polymers. Not to mention the reversed phase chromatography that shows numerous applications in every analytical field.

Moreover comprehensive-two dimensional 2D chromatography can allow the separation of compounds with similar or identical properties in mixtures or in various matrices by increasing peak capacity resolution.

Miniaturization trend in modern HPLC instrumentation is reflected to Micro, Nano, Capillary or Ultra high performance liquid chromatography. The latter was the great revolution in liquid chromatography that occurred, with significant advances in instrumentation and column technology, with outstanding improvement in resolution, speed and sensitivity.

Evidently, recent advances in analytical methodologies take into consideration the Green Chemistry perspectives, thus using less hazardous, green solvents, or no solvent less approaches. Any analytical chemist when developing a new protocol should bare in mind the twelve principles of Green Analytical Chemistry as proposed by Jacek Namieśnik.

Of course no matter how sophisticated hardware is used in the analytical method, sample preparation will always be the bottleneck as it is the most determinative step, at least until a universal and ideal technique is discovered. Automation is now more mandatory than ever. Trends in sample preparation techniques are represented by micro-extraction techniques including On-line Solid Phase Extraction (SPE) Microwave Assisted Solvent Extraction (MASE), Solid Phase Microextraction (SPME), Supercritical Fluid Extraction (SFE), Single Drop Micro Extraction, (SDME), Fabric Phase Sorptive Extraction (FPSE), Stir Bar Sorptive Extraction (SBSE) and others, all promising in reducing solvent consumption. New materials of nanostructures, such as graphene, carbon nanotubes, biomaterials, immunoaffinity materials are also introduced in the sample pretreatment step either for clean-up or for isolation. All above mentioned techniques comply with green analytical chemistry demands and ensure environmental protection and public safety. Applied prior to HPLC offer the necessary selectivity, sensitivity and lower detection required to meet the legislation criteria and give a solution to any analytical problem.

\section{Concluding remarks}

Speed, sample throughput and selectivity are the driving forces in the selection of the ideal analytical tool in the analytical laboratory. HPLC offers a variety of versions and thus it is the separation technique of choice with a wide range of applications. Therefore it is a powerful tool fully adapted by most analytical chemists and is currently the state-of-the art in separation science. Reasonably the advances in hardware manufacture rendered HPLC from workhorse, to the racehorse in the lab.

\section{References}

1. Anastas PT. Green Chemistry and the role of analytical methodology development. CRITICAL REVIEWS IN ANALYTICAL CHEMISTRY. 1997;29(3):167-175.

2. Kabir A, Kenneth G. Furton, Abdul Malik. Innovations in sol- 
gel microextraction phases for solvent-free sample preparation in analytical chemistry. TrAC Trends in Analytical Chemistry. 2013;45:197-218.

3. Jacek Namieśnik. Trends in environmental analytics and monitoring Critical Reviews in Analytical Chemistry. 2000;30(2-3):221-269.

4. Papadoyannis I, Samanidou V. Ion chromatography principles suppressed and non-suppressed. Marcel Dekker. Jack Cazes Ed. Published on line, 2009.

5. Papadoyannis I, Samanidou V. Ion Exclusion Chromatography. Marcel Dekker. Jack Cazes Ed. Published on line, 2009.

6. Papadoyannis I, Samanidou V. Sample Preparation Prior to HPLC
Marcel Dekker. Jack Cazes Ed. Published on line, 2009.

7. Samanidou V, Karageorgou E, Kourti P. "The role of HPLC in the authenticity control of milk and dairy products". Edited collection Liquid Chromatography: Principles, Technology and Applications. Nova Publishers. 2013;173-206.

8. Samanidou V, Papadoyannis I. HPLC: the dominant separation technique with a wide range of applications. Edited collection."Chromatography: Types, Techniques and Methods."Nova Publishers. Ed. Frank Columbus. 2009. ISBN: 978-1-60876-316-0.

9. Samanidou VF, Nika MK, Papadoyannis IN. HPLC as a tool in medicinal chemistry for the monitoring of tricyclic antidepressants in biofluids. Mini Rev Med Chem. 2008;8(3):256-75. 